\title{
Spindle assembly in the absence of chromosomes in mouse oocytes
}

\author{
Ji-Wen Yang ${ }^{1,2}$, Zi-Li Lei ${ }^{1,2}$, Yi-Liang Miao ${ }^{1,2}$, Jun-Cheng Huang ${ }^{1,2}$, Li-Hong Shi, ${ }^{1,2}$ \\ Ying-Chun OuYang ${ }^{1}$, Qing-Yuan Sun ${ }^{1}$ and Da-Yuan Chen ${ }^{1}$ \\ ${ }^{1}$ State Key Laboratory of Reproductive Biology, Institute of Zoology, Chinese Academy of Sciences, \# 5 Da Tun Road, \\ Chaoyang, Beijing 100101, People's Republic of China and ${ }^{2}$ Graduate School, Chinese Academy of Sciences, Beijing \\ 100039, People's Republic of China
}

Correspondence should be addressed to D-Y Chen; Email: chendy@ioz.ac.cn

\begin{abstract}
This study was carried out to investigate the contributions of chromosomes to spindle assembly in mouse oocytes. We generated two groups of cytoplasts (holo- and hemi-cytoplasts) by enucleation of germinal vesicle (GV), metaphase I (MI), and metaphase II (MII) oocytes using micromanipulation technology. After in vitro culture for $18 \mathrm{~h}$, spindles with different shapes (bi-, mono-, or multipolar) formed in most of these cytoplasts except in hemi-GV cytoplasts. Two or more spindles were observed in most of holo-GV, holo-MI, and holo-MII cytoplasts (76.1, 77.0, and $83.7 \%$ respectively). However, the proportions of hemi-MI and hemi-MII cytoplasts with multiple sets of spindles decreased to $\mathbf{1 7 . 6}$ and $\mathbf{2 0 . 7} \%$ respectively. A single bipolar spindle was observed in each sham-operated oocyte generated by removing different volumes of cytoplasm from the oocytes and keeping nuclei intact. Localization of $\gamma$-tubulin showed that microtubule organizing centers (MTOCs) were dispersed at each pole of the multiple sets of spindles formed in holo-cytoplasts. However, most of the MTOCs aggregated at the two poles of the bipolar spindle in sham-operated oocytes. Our results demonstrate that chromosomes are not essential for initiating spindle assembly but for directing distinct MTOCs to aggregate to form a bipolar spindle. Some factors of undetermined nature may pre-exist in an inactive form in GV-stage ooplasm, serving as initiators of spindle assembly upon their activation. Moreover, GV materials released into the cytoplasm may facilitate spindle assembly in normal meiotic maturation.
\end{abstract}

Reproduction (2007) 134 731-738

\section{Introduction}

The precise segregation of chromosomes in mitosis and meiosis is driven by a fusiform structure called the spindle, which is a complex microtubule-based structure. In the spindle, the minus ends of microtubules are at or near the poles and plus ends are at the equator of the spindle (Compton 2000, Karsenti \& Vernos 2001, Mitchison \& Salmon 2001, Winey \& O'Toole 2001). Spindle assembly and function are intimately linked to the intrinsic dynamics of microtubules. In most animal somatic cells, microtubules are nucleated at centrosomes, which are composed of a pair of perpendicularly oriented centrioles and numerous surrounding proteins called pericentriolar material (PCM). The centrosome is considered as the microtubule organizing center (MTOC). These microtubules are then captured and stabilized by kinetochores on chromosomes, followed by the formation of a bipolar spindle. However, in oocytes of many animal species (Szollosi et al. 1972, Steffen et al. 1986, McKim \& Hawley 1995, de Saint Phalle \& Sullivan 1998) and plant cells (Smirnova \&
Bajer 1992), which do not possess centrosomes, spindle assembly occurs via an acentrosomal pathway. In these cells, even in the absence of centrioles, electron-dense materials can be observed using anti-PCM antibodies (Calarco-Gillam et al. 1983). These materials are commonly considered as MTOCs. It has been found that $\gamma$-tubulin, a highly conserved member of the tubulin superfamily, is localized at MTOCs (Gueth-Hallonet et al. 1993) and functions in microtubule nucleation (Zheng et al. 1995). Fully grown mammalian oocytes are arrested at the diplotene stage of the first meiotic prophase, which is also termed the germinal vesicle (GV) stage. At this stage, the multiple MTOCs are already dispersed in the cytoplasm (Van Cauwenberge \& Alexandre 1992). Microtubules nucleated by these MTOCs radiate throughout the cytoplasm. After GV breakdown (GVBD) followed by the mixing between the GV materials and the cytoplasm, the chromosomes are exposed to the cytoplasm, and microtubules nucleated from MTOCs form multiple cytoplasmic asters. Rapid and dynamic microtubule reorganizations take place by 
the interactions between cytoplasmic asters and chromosomes. Finally, the metaphase I (MI) bipolar spindle is organized from microtubules through the action of motor proteins, and the two spindle poles result from pre-existing cytoplasmic MTOCs. In some cases, monopolar or multipolar spindle-like structures assemble under abnormal conditions (Verlhac et al. 1993, Compton 2000, Meng et al. 2004). Then, the MI spindle initially positioned at the oocyte's center migrates to the cortex. After emission of the first polar body, oocytes are arrested at metaphase II (MII) stage with the MII spindle located beneath the plasma membrane (Calarco et al. 1972, Maro et al. 1985).

As described previously, spindle assembly starts after GVBD. However, it is not clear which factors induce or initiate this process in mouse oocytes. Several studies have been conducted to investigate the contributions of chromosomes to initiating spindle assembly. In grasshopper spermatocytes, spindle assembly is promptly initiated upon premature exposure of centrosomes to chromosomes by rupturing the nuclear envelope during prophase, but no spindle forms if all chromosomes are removed from the nucleus before the nuclear envelope breaks down (Zhang \& Nicklas 1995). This result agrees with the findings in Xenopus eggs in which centrosomes co-injected with demembranated nuclei are activated only when a nucleus is nearby (Karsenti et al. 1984). The above results support the proposition that chromosomes are the activators of spindle assembly. However, Drosophila secondary spermatocytes can assemble morphologically normal spindles in the absence of chromosomes (Bucciarelli et al. 2003). These results suggest that the importance of chromosomes in starting spindle formation varies in different kinds of cells and/or different species. To our knowledge, there is only one report using anucleated mouse oocytes that have no centrioles as a model to investigate the impact of chromosomes on spindle assembly; spindles are formed in anucleated fragments of $\mathrm{MI}$ and MII oocytes derived by bisecting oocytes (Brunet et al. 1998). However, this study cannot answer the question whether chromosomes are necessary for initiating spindle assembly, because the above bisection is performed after GVBD, by which time the chromosomes have already been exposed to the cytoplasm, and have probably changed the cytoplasmic property before they are removed (Karsenti \& Vernos 2001). Thus, it is not quite clear whether chromosomes play a critical role in initiating spindle assembly in mouse oocytes. Furthermore, in the above study, single bipolar spindles can assemble in most of anucleated fragments of MII oocytes. It appears that dispersed MTOCs can interact with each other and aggregate to form a single spindle without any directions from chromosomes. However, anucleated fragments of oocytes generated by bisection possess only half of the original cytoplast volume. In these half-sized cytoplasts (referred to as 'hemi-cytoplasts' in the present study), the distance between dispersed MTOCs is relatively short and the chances for them to interact with each other are likely to be increased. In contrast, normal-sized cytoplasts (referred to as 'holo-cytoplasts' in the present study) derived by enucleation of oocytes with little cytoplasm possess their original cytoplast volume. The distance between dispersed MTOCs in these cytoplasts is relatively long and the chances for them to interact with each other are likely to be reduced. Thus, another question is whether dispersed MTOCs can interact with each other and aggregate to form single bipolar spindles in normal-sized cytoplasts in the absence of chromosomes. To address these questions, we generated two groups of cytoplasts (hemi- and holo-cytoplasts) by micromanipulation from GV, MI, and MII oocytes in which spindle assembly was assessed. Some oocytes that had been manipulated in the same manner to remove the same volume of cytoplasm without removing the nuclei were used as control and referred to 'sham-operated oocytes' in this study.

\section{Results \\ Spindle assembly in sham-operated oocytes and anucleated cytoplasts after culture for $18 \mathrm{~h}$}

As summarized in Table 1, a single bipolar spindle was observed in each sham-operated oocyte after in vitro culture. No monopolar or multipolar spindles formed in these oocytes. All of the bipolar spindles in hemi-oocytes were apparently smaller than those in holo-oocytes (Fig. $1 \mathrm{~A}$ and $\mathrm{B}$ ).

Table 1 Spindle assembly in sham-operated GV, MI and MII mouse oocytes after culture for $18 \mathrm{~h}$.

No. of oocytes with different types of spindles (\%)

\begin{tabular}{|c|c|c|c|c|c|c|}
\hline \multirow[b]{2}{*}{ Type of oocytes } & \multirow[b]{2}{*}{ Replicates } & \multirow[b]{2}{*}{ No. of oocytes } & & \multirow[b]{2}{*}{$\begin{array}{l}\text { No. of oocytes } \\
\text { with multiple sets } \\
\text { of spindles }(\%)^{\mathrm{a}}\end{array}$} \\
\hline & & & Bipolar spindles & Monopolar spindles & Multipolar spindles & \\
\hline Hemi-GV oocytes & 3 & 42 & $42(100)$ & $0(0)$ & $0(0)$ & $0(0)^{*}$ \\
\hline Holo-GV oocytes & 4 & 55 & $55(100)$ & $0(0)$ & $0(0)$ & $0(0)^{*}$ \\
\hline Hemi-MI oocytes & 3 & 34 & $34(100)$ & $0(0)$ & $0(0)$ & $0(0)^{*}$ \\
\hline Holo-MI oocytes & 4 & 45 & $45(100)$ & $0(0)$ & $0(0)$ & $0(0)^{*}$ \\
\hline Hemi-MII oocytes & 3 & 31 & $31(100)$ & $0(0)$ & $0(0)$ & $0(0)^{*}$ \\
\hline Holo-MII oocytes & 3 & 33 & $33(100)$ & $0(0)$ & $0(0)$ & $0(0)^{*}$ \\
\hline
\end{tabular}

*Values with same superscripts within a column do not differ significantly $(P>0.05)$. GV, germinal vesicle; MI, metaphase I; MII, metaphase II

${ }^{\mathrm{a} O o c y t e s ~ h a v i n g ~ m o r e ~ t h a n ~ o n e ~ s p i n d l e ~ i n s i d e ~ w e r e ~ c o n s i d e r e d ~ t o ~ h a v e ~ m u l t i p l e ~ s e t s ~ o f ~ s p i n d l e s . ~}$ 

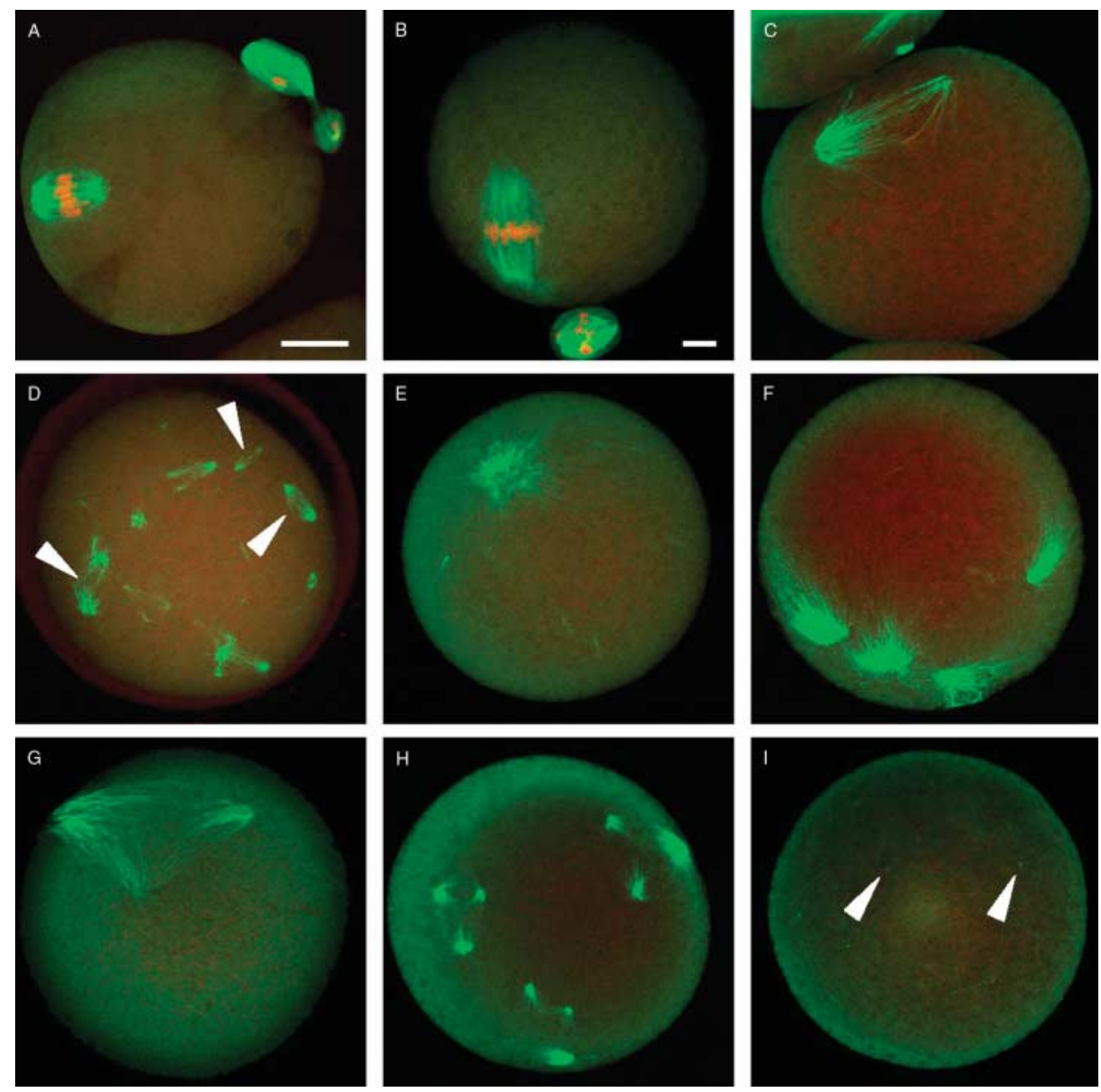

Figure 1 Microtubule organization in sham-operated oocytes and anucleated cytoplasts $18 \mathrm{~h}$ after manipulation. (A) A single bipolar spindle in shamoperated hemi-oocyte. (B) A single bipolar spindle in sham-operated holooocyte. (C) A single bipolar spindle in an anucleated hemi-cytoplast.

(D) Multiple sets of bipolar spindles in an anucleated holo-cytoplast. Triangles indicate multiple sets of bipolar spindles which are smaller in size than normal bipolar spindles. (E) A single monopolar spindle in an anucleated hemi-cytoplast. (F) Multiple sets of monopolar spindles in an anucleated holo-cytoplast. (G) A single multipolar spindle in an anucleated hemi-cytoplast. (H) Three multipolar spindles in an anucleated holo-cytoplast. (I) No spindles were detectable in anucleated hemi-GV cytoplast. Triangles indicate small aster-like structures. Microtubules were stained by FITC-conjugated mouse anti- $\alpha$-tubulin antibody (green) and chromosomes by propidium iodide (red). Bar $=10 \mu \mathrm{m}$.
Unlike what happened in sham-operated oocytes, not only bipolar spindles but also monopolar and multipolar spindles were observed in anucleated cytoplasts (Table 2, Fig. 1C-H). Two or more spindles were observed for each cytoplast in most of the holo-GV, holo-MI, and holo-MII cytoplasts $(76.1,77.0$, and $83.7 \%$ respectively). However, the proportion of hemi-MI and hemi-MII cytoplasts with multiple sets of spindles decreased to 17.6 and $20.7 \%$ respectively (Table 2). Interestingly, unlike the cases in holo-GV cytoplasts, no spindles were observed in hemiGV cytoplasts. Only a few small aster-like structures were detectable in these cytoplasts. In some cases, these asterlike structures were connected by extremely thin microtubule bundles (Table 2, Fig. 1I). All spindles observed in anucleated cytoplasts were apparently smaller in size than those observed in sham-operated holo-oocytes (Fig. 1A-H). All the results are summarized in Fig. 4.

\section{Localization of $\gamma$-tubulin in sham-operated oocytes and anucleated cytoplasts after culture for $18 \mathrm{~h}$}

$\gamma$-Tubulin is an important component of MTOCs. Thus, the localization of MTOCs was accomplished by using $\gamma$-tubulin as a marker. In sham-operated oocytes, $\gamma$-tubulin aggregated at the bipolar spindle poles.
Some distinct $\gamma$-tubulin foci were also detected in the cytoplasm (Fig. 2A). However, in holo-cytoplasts with the assembly of multiple sets of spindles, $\gamma$-tubulin foci were found at each pole of these spindles, either bipolar or tripolar (Fig. 2B). Similarly, $\gamma$-tubulin foci were found at the poles of monopolar spindles in hemi-cytoplasts (Fig. 2C).

\section{Discussion}

In the present study, spindle-shaped microtubule arrays formed in holo-GV cytoplasts, indicating that chromosomes are not essential for initiating spindle assembly, and the initial spindle assembly occurs in a chromosome-independent pathway in mouse oocytes. Several studies (Theurkauf \& Hawley 1992, Afshar et al. 1995, Murphy \& Karpen 1995, Vernos et al. 1995, Wang \& Adler 1995) have indicated that the nod family of kinesin-related proteins is involved in initiating microtubule reorganization. These proteins bind tightly to DNA and have plus end-directed motility. Perturbation of the function of these proteins reduces the efficiency of spindle assembly and causes a poor association between the chromosomes and the spindle. Nevertheless, the function of these proteins in mammalian cells has not 
Table 2 Spindle assembly in anucleated GV, MI and MII cytoplasts after culture for $18 \mathrm{~h}$.

No. of cytoplasts with different types of spindles (\%)

\begin{tabular}{lcccccc} 
Type of cytoplasts & Replicates & No. of cytoplasts & Bipolar spindles & Monopolar spindles & Multipolar spindles & $\begin{array}{c}\text { multiple sets of } \\
\text { spindles }(\%)^{\text {a }}\end{array}$ \\
\hline Hemi-GV cytoplasts & 3 & 55 & $0(0)$ & $0(0)$ & $0(0)$ & $0(0)^{\ddagger}$ \\
Holo-GV cytoplasts & 3 & 67 & $34(50.7)$ & $15(22.4)$ & $18(26.9)$ & $51(76.1)^{*}$ \\
Hemi-Ml cytoplasts & 3 & 68 & $41(60.3)$ & $22(32.4)$ & $5(7.3)$ & $12(17.6)^{\dagger}$ \\
Holo-MI cytoplasts & 4 & 74 & $26(35.1)$ & $42(56.8)$ & $6(8.1)$ & $57(77.0)^{*}$ \\
Hemi-MII cytoplasts & 3 & 58 & $33(56.9)$ & $19(32.8)$ & $6(10.3)$ & $12(20.7)^{+}$ \\
Holo-MII cytoplasts & 3 & 43 & $10(23.3)$ & $22(51.2)$ & $11(25.5)$ & $36(83.7)^{*}$ \\
\hline
\end{tabular}

*, †, fValues with different superscripts within a column differ significantly $(P<0.05)$. GV, germinal vesicle; MI, metaphase I; MII, metaphase II. ${ }^{a}$ Oocytes having more than one spindle inside were considered to have multiple sets of spindles.

been examined. In the present study, spindle assembly occurred in the absence of chromosomes, and thus in the absence of the above DNA-binding proteins. The result that holo-GV cytoplasts assembled spindle-like structures in this study suggests that there are some other factors of undetermined nature serving as initiators

$\alpha$-tubulin
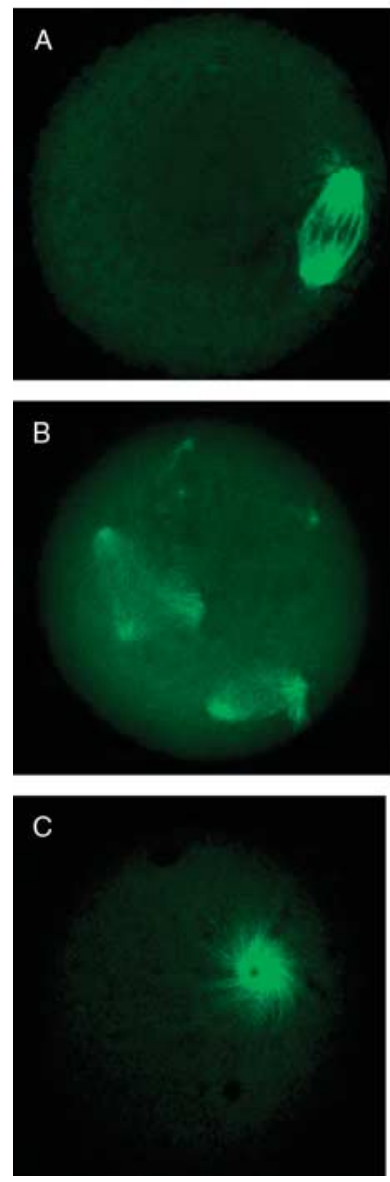

$\gamma$-tubulin
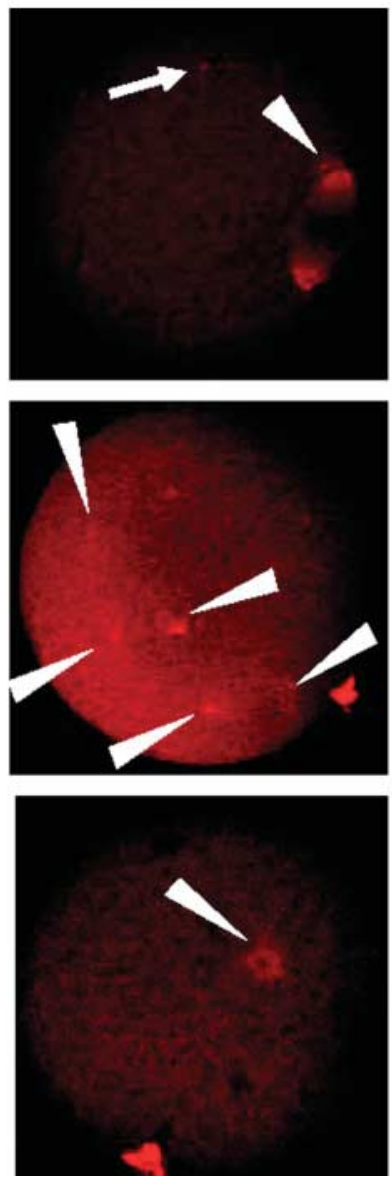

of spindle assembly and these factors pre-exist in GV-stage oocyte cytoplasm before GVBD. They may be suppressed in an inactive form in the prophase oocyte cytoplasm. How are these factors activated in the absence of chromosomes in the GV cytoplast during in vitro culture? Schultz et al. (1978) have shown that
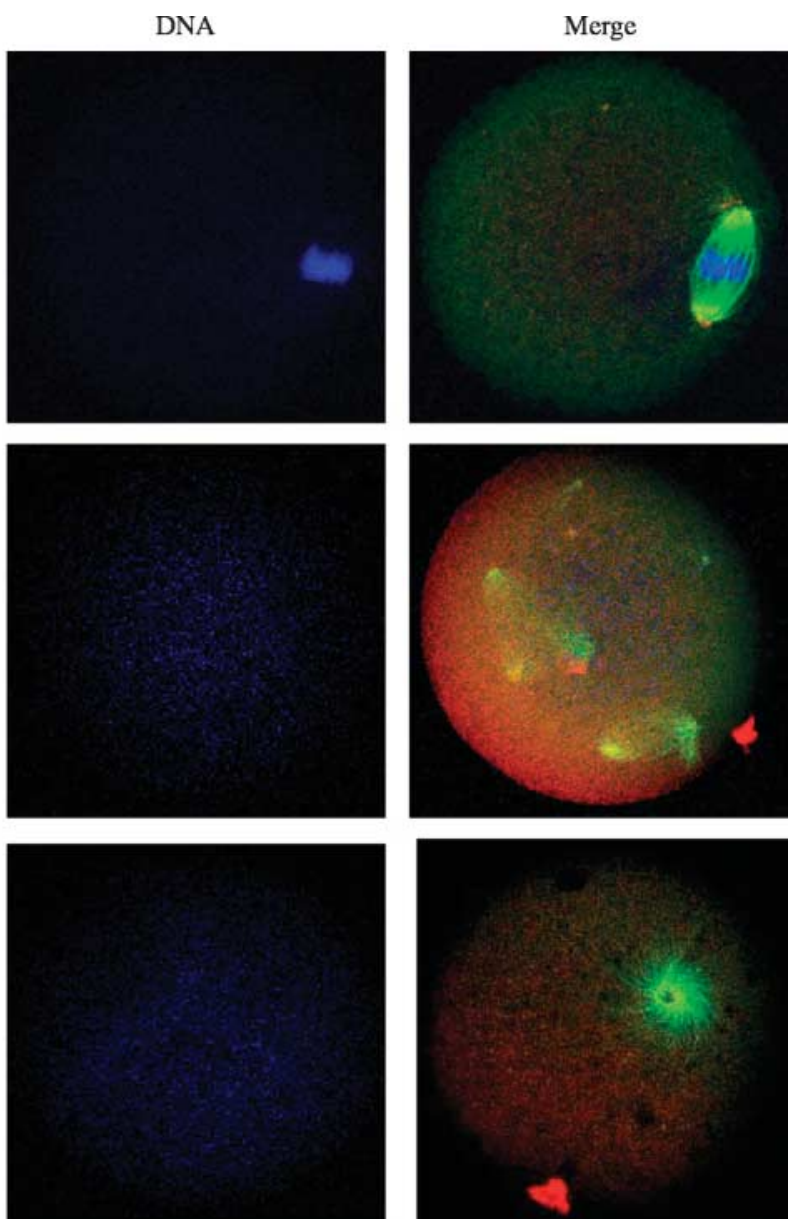

Figure 2 Localization of $\gamma$-tubulin in sham-operated mouse oocytes and anucleated cytoplasts after culture for $18 \mathrm{~h}$. Green, $\boldsymbol{\alpha}$-tubulin; red, $\gamma$-tubulin; blue, chromosome; yellow, overlapping of $\alpha$ - and $\gamma$-tubulins. (A) $\gamma$-Tubulin aggregated the bipolar spindle poles in a sham-operated oocyte. The white triangle indicates $\gamma$-tubulin foci at one pole of the bipolar spindle. Some distinct $\gamma$-tubulin foci were also detected in the cytoplasm. The white arrow indicates one of these distinct foci in the cytoplasm. (B) In the holo-cytoplast, $\gamma$-tubulin foci were dispersed at each pole of multiple sets of spindles. The white triangle indicates $\gamma$-tubulin foci at each pole of the multipolar and bipolar spindle. (C) In the hemi-cytoplast, $\gamma$-tubulin foci were detected at the pole of the monopolar spindle. The white triangle indicates $\gamma$-tubulin foci at the pole of the monopolar spindle. 
many changes in protein synthesis normally associated with meiotic maturation of mouse oocytes also occur in anucleated GV oocyte fragments in the absence of nuclear progression, and the reprogramming of protein synthesis during meiotic maturation is directed by RNA templates already present in the cytoplasm. According to this finding, we hypothesize that the factors responsible for starting spindle assembly are activated in the GV cytoplast during in vitro culture. They then stabilize microtubules and start spindle assembly. Clearly, further investigations are needed to fully understand these factors and also their upstream activators.

Do these factors work alone to initiate spindle assembly? There are some differences in the cytoplasmic property between GV and MI (or MII) cytoplasts. The GV cytoplasts were derived by removing the whole GV before GVBD to avoid the mixing between the GV material and the cytoplasm, and also to avoid exposing chromosomes to the cytoplasm. In MI or MII cytoplasts, which were generated after GVBD, GV materials have been released in the cytoplasm, and they are essential for subsequent development (Katagiri \& Moriya 1976, Balakier \& Tarkowski 1980). In the present study, interestingly, unlike what happened in holo-GV cytoplasts, no spindles formed in hemi-GV cytoplasts. Only several small aster-like structures were detectable (Fig. 1I). However, spindle assembly occurred in hemi-MI and hemi-MII cytoplasts. One possible explanation is that in holo-GV cytoplasts, factors responsible for initiating spindle assembly may concentrate to initiate spindle assembly, while in hemi-GV cytoplasts, the concentration of these factors in a local area is below the threshold level necessary for their normal function, resulting in the failure of spindle formation. However, in MI or MII cytoplasts, GV materials have been released to the cytoplasm after GVBD and are likely to facilitate the initial spindle assembly.

What, then, is the role of chromosomes in spindle assembly in mouse oocytes? In this study, a single bipolar spindle formed in each sham-operated oocyte. Localization of MTOCs using $\gamma$-tubulin as a marker showed that MTOCs aggregated at the two poles of the bipolar spindle in sham-operated oocytes. However, in holo-cytoplasts, multiple sets of spindles formed in most cases. MTOCs were dispersed at each pole of these spindles. The above results suggest that although spindle assembly can be initiated in the absence of chromosomes in normal-sized cytoplasts, MTOCs dispersed throughout the cytoplasm can only interact with other adjacent ones. Local interactions between MTOCs result in the formation of several spindleshaped arrays, which seem to lose the ability to aggregate in the absence of chromosomes. Thus, chromosomes play a critical role in organizing these scattered spindle-shaped arrays into single bipolar spindles. It seems that chromosomes could locally modify the cytoplasm state to favor spindle formation (Sun et al. 2001). Support also comes from observations of microtubule assembly around chromatin or chromosomes (Karsenti et al. 1984, Dogterom et al. 1996) and chromosome micromanipulation experiments (Nicklas \& Gordon 1985). Chromosomes are likely to act as the directors during spindle assembly. Several studies have suggested that chromatin-bound Ran-GEF, RCC1, can catalyze the Ran-GDP/Ran-GTP transition, generating a high local concentration of Ran-GTP around chromosomes that stimulates MT growth and stability. However, the high activity of Ran-GTPase-activating protein (Ran-GAP), which is cytoplasmic, can catalyze the Ran-GTP/Ran-GDP transition and reduce the concentration of Ran-GTP in the cytoplasm (Carazo- Salas et al. 1999, 2001, Caudron et al. 2005). Thus, it is thought that a dynamics gradient generated by chromosomes exists in the cytoplasm, which directs microtubules to reorganize into a normal-sized bipolar spindle (Karsenti \& Vernos 2001). In the absence of chromosomes, the cytoplasmic state is uniform, and spindle assembly occurs in disorder. However, Dumont et al. (2007) found that manipulating Ran-GTP levels did not inhibit assembly of functional meiosis I spindles in living mouse oocytes. Further investigations are needed to clarify this point. In the present study, dispersed MTOCs did not aggregate in the absence of chromosomes, demonstrating that chromosomes provide cues for correct bipolar spindle assembly, which is also supported by a recent study (Gaetz et al. 2006). Moreover, monopolar and multipolar spindles formed in cytoplasts devoid of chromosomes, indicating that asters dispersed in the cytoplasm interact with each other in a rather random way without the direction of chromosomes.

In this study, some distinct $\gamma$-tubulin foci were also detected in the cytoplasm of sham-operated oocytes. This result is similar to that obtained by previous studies (Gueth-Hallonet et al. 1993, Palacios et al. 1993, Meng et al. 2004). In contrast, no additional $\gamma$-tubulin foci were detected in both hemi- and holocytoplasts besides their localization to spindle poles and asters. It is possible that numerous MTOCs have been removed from the cytoplasm during enucleation, and there are fewer MTOCs in hemi-cytoplasts than those in holo-cytoplasts. This may explain why the proportion of anucleated cytoplasts with multiple sets of spindles in holo-group was much higher than that in hemi-group.

In summary, our results suggest that chromosomes are not essential for initiating spindle assembly but for directing distinct MTOCs to aggregate into a bipolar spindle in mouse oocytes. Some unknown factors responsible for initiating microtubule reorganization pre-exist in GV oocyte cytoplasm. After being activated during meiotic maturation, they stabilize microtubules to start spindle formation. Further investigations are needed to study the biochemical nature of these factors 
and the way they function. Moreover, GV materials released into the cytoplasm may facilitate spindle assembly in the normal meiotic maturation process. However, it should be stressed that all the results obtained in this study relate only to the mouse oocyte system and may vary in different species.

\section{Materials and Methods}

\section{Animals}

Female Kunming mice were housed in a temperature- and light-controlled room with free access to water and food. Illumination was on between 0600 and $1800 \mathrm{~h}$. The experimental protocols were approved by the ethical committee of Institute of Zoology, Chinese Academy of Sciences.

\section{Preparation of GV-MI-, and MII-stage oocytes}

Immature GV oocytes were retrieved by puncturing the follicles of each ovary dissected from 6- to 8-week-old female mice $48 \mathrm{~h}$ after injection of $10 \mathrm{IU}$ pregnant mare serum gonadotropin (PMSG; Tianjin Animal Hormone Factory). The oocytes were stripped of cumulus cells by repeated aspiration through the tip of a hand-drawn pipette. In order to prevent GVBD and to help oocytes develop a perivitelline space, the cumulus-denuded GV oocytes were then transferred into human tubal fluid (HTF) medium supplemented with $10 \%$ (v/v) fetal calf serum (FCS; HyClone, Logan, UT, USA) and $50 \mu \mathrm{g} / \mathrm{ml}$ 3-isobutyl-1-methylxanthine (IBMX; Sigma Chemical Co.) and cultured for $2 \mathrm{~h}$ in $5 \% \mathrm{CO}_{2}$ in air at $37^{\circ} \mathrm{C}$. The oocytes with visible GVs and perivitelline spaces were selected for enucleation. MI oocytes were obtained by culturing GV-stage oocytes in HTF medium supplemented with $10 \%$ FCS $(\mathrm{v} / \mathrm{v})$ for $8 \mathrm{~h}$.

For the collection of MIl oocytes, each female mouse was injected i.p. with $10 \mathrm{IU}$ PMSG. After $48 \mathrm{~h}, 10 \mathrm{IU}$ human chorionic gonadotropin (hCG; Ningbo Animal Hormone Factory, Ningbo, Zhejiang, China) were administered to induce ovulation. After $14 \mathrm{~h}$, cumulus-oocyte complexes were collected from oviducts. Cumulus cells were removed with $300 \mathrm{IU} / \mathrm{ml}$ hyaluronidase (Sigma).

\section{Enucleation of oocytes}

Enucleation of GV-stage oocytes was conducted as described by Takeuchi etal. (1999) and Chang et al. (2004). Some modifications were included. Briefly, the GV-stage oocytes were transferred in M2 medium (Sigma) with $25 \mu \mathrm{g} / \mathrm{ml} \mathrm{IBMX}$ and $10 \mu \mathrm{g} / \mathrm{ml}$ cytochalasin B (Sigma), and then the zona pellucida of each oocyte was lanced with a sharp needle. The GV was removed using a micropipette with a $15 \mu \mathrm{m}$ inner diameter (Fig. 3A and B).

The enucleation of MI or MII oocytes was performed in M2 medium with $7.5 \mu \mathrm{g} / \mathrm{ml}$ cytochalasin B. The MI or MII spindle appeared transparent in the ooplasm. After lancing of the zona pellucida with a microneedle, MI or MII karyoplast was removed also using a micropipette with a $15 \mu \mathrm{m}$ inner diameter (Fig. 3C-F).
After enucleation, all of the manipulated oocytes were cultured in HTF medium supplemented with $10 \%$ (v/v) FCS in $5 \%(\mathrm{v} / \mathrm{v}) \mathrm{CO}_{2}$ in air at $37^{\circ} \mathrm{C}$ for $18 \mathrm{~h}$ before immunohistochemistry analysis.

\section{Immunofluorescence and laser scanning confocal microscopy}

In the present study, the localization of microtubules and MTOCs was examined by staining of $\alpha$ - and $\gamma$-tubulins respectively. To visualize microtubules, cytoplasts or oocytes were fixed in $4 \%(\mathrm{w} / \mathrm{v})$ paraformaldehyde in PBS for $30 \mathrm{~min}$,
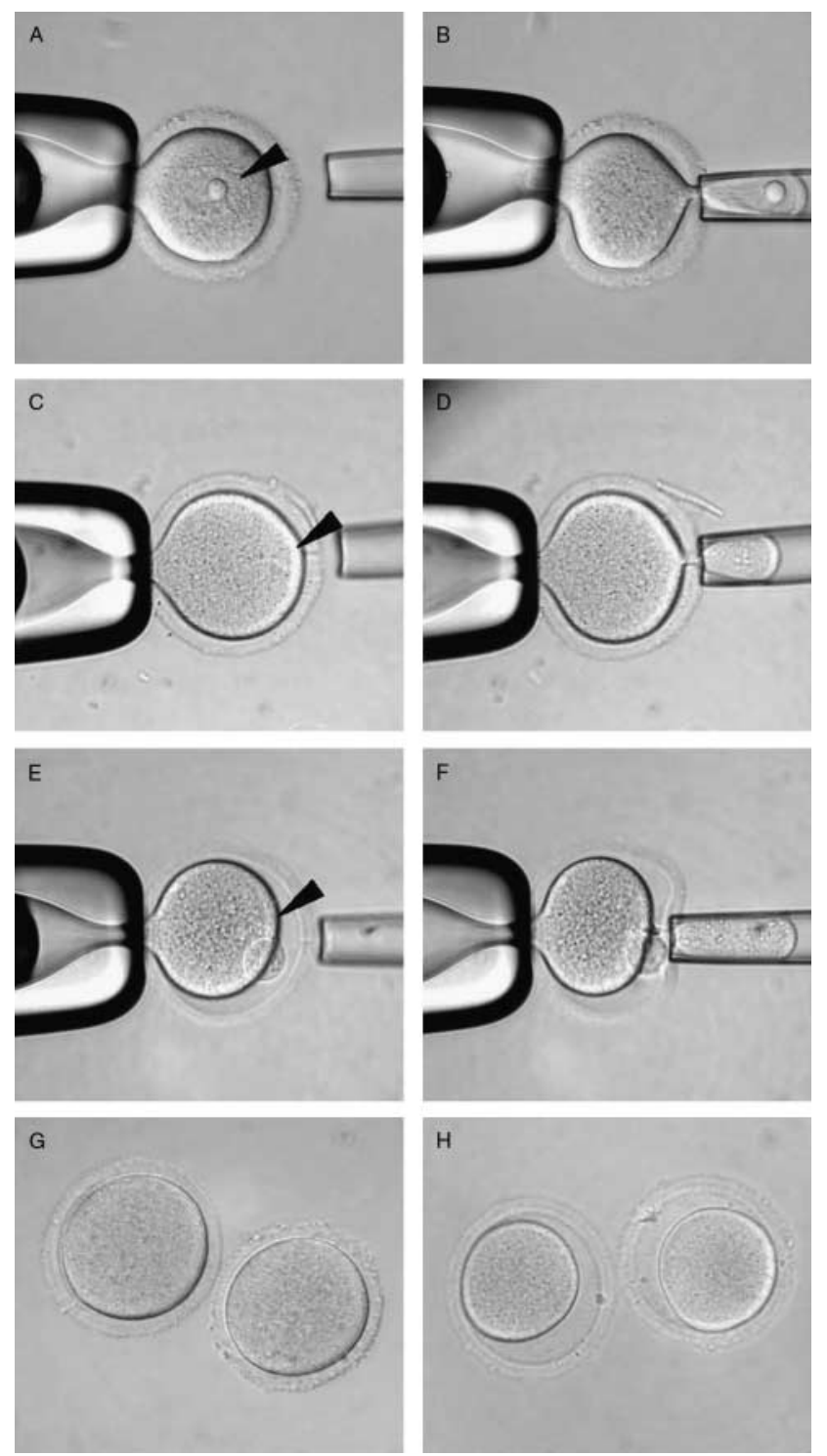

Figure 3 Preparation of cytoplasts from GV-, MI- and MII-stage mouse oocytes. (A and B) Removal of GV from a GV-stage oocyte. (C and D) Removal of MI spindle from a MI-stage oocyte. (E and F) Removal of MII spindle from a MII-stage oocyte. (G) Holo-cytoplast generated by removing the nucleus with minimum cytoplasm from an oocyte. $(\mathrm{H})$ Hemi-cytoplast with half of the original oocyte volume. Triangles indicate nuclei of GV-, MI- and MII-stage oocytes. 


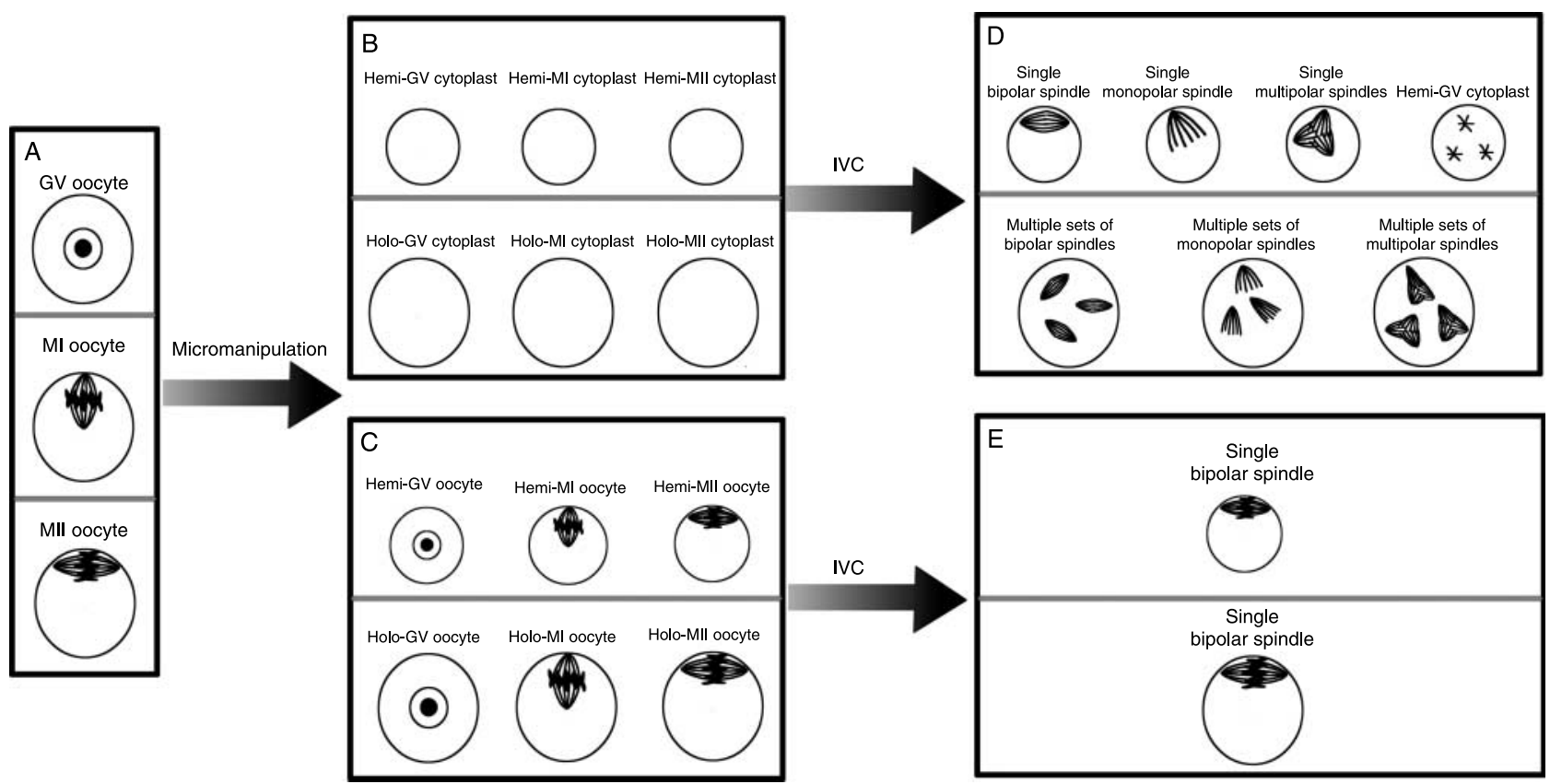

Figure 4 Summary of experimental designs and results. (A) Oocytes at three meiotic stages: GV (germinal vesicle), MI (metaphase I), and MII (metaphase II) oocytes. (B) Two groups of cytoplasts with different volume of cytoplasm were derived from enucleation of oocytes: hemi-cytoplasts possessed only half of the original cytoplast volume and holo-cytoplasts were generated with little cytoplasm removal and possessed original cytoplast volume. (C) Two groups of sham-oocytes with different volumes of cytoplasm removed: hemi-oocytes possessed only half of the original oocyte volume, and holo-oocytes with little cytoplasm removed possessed original oocyte volume. (D) After in vitro culture (IVC), spindle-shaped microtubule arrays with different shapes (bipolar, monopolar, or multipolar) formed in all kinds of cytoplasts except hemi-GV cytoplasts, in which only a few small aster-like structures were detectable; the proportion of cytoplasts with multiple sets of spindles in holo-cytoplast group was much higher than that in hemi-cytoplast group. (E) Single bipolar spindles formed in both hemi- and holo-oocytes, in which chromosomes were contained.

and permeabilized for $30 \mathrm{~min}$ in PBS buffer containing $0.5 \%$ $(\mathrm{v} / \mathrm{v})$ Triton $\mathrm{X}-100$, and then incubated in PBS buffer with $1 \%$ $(\mathrm{w} / \mathrm{v})$ BSA for $1 \mathrm{~h}$. After incubation with 1:100 fluorescein isothiocyanate (FITC)-conjugated mouse anti- $\alpha$-tubulin antibody for $1 \mathrm{~h}$, the cytoplasts or oocytes were washed in PBS with $0.1 \%(\mathrm{v} / \mathrm{v})$ Tween 20 and $0.01 \%(\mathrm{v} / \mathrm{v})$ Triton X-100 thrice. DNA was stained by exposing the cytoplast or oocytes to $10 \mu \mathrm{g} / \mathrm{ml}$ propidium iodide (Sigma) to check the absence of chromosomes. All the above procedures were performed at room temperature. Finally, the oocytes were mounted on glass slides and examined using a TCS-4D laser scanning confocal microscope (Leica Microsystems).

When cytoplasts or oocytes were double labeled for $\alpha$ - and $\gamma$-tubulins, they were incubated with 1:200 mouse anti- $\gamma$ tubulin antibody for $1 \mathrm{~h}$ before three washes, and then incubated with 1:100 tetramethyl rhodamine iso-thiocyanate (TRITC)-conjugated goat anti-mouse IgG for $1 \mathrm{~h}$. After another three washes, the cytoplasts or oocytes were incubated with 1:100 FITC-conjugated mouse anti- $\alpha$-tubulin antibody for $1 \mathrm{~h}$. Chromosomes were then stained with Hoechst 33258 $(10 \mu \mathrm{g} / \mathrm{ml}$ in PBS) for $40 \mathrm{~min}$. Finally, cytoplasts or oocytes were mounted on glass slides and examined.

\section{Experimental design}

Two groups were generated to evaluate the capacity of cytoplasts in whole or half of the original cytoplast volume to assemble spindles in the absence of chromosomes. In each group, all three types of oocytes (GV, MI, and MII oocytes) were used to investigate the effect of GVBD on spindle assembly. For the first group (hemi-cytoplasts), nuclei were removed with cytoplasm in half of the original oocytes volume (Fig. $3 \mathrm{H}$ ). Some oocytes that had been manipulated in the same manner to remove the same volume of cytoplasm without removing the nuclei were used as sham-operated oocytes (hemi-oocytes). In the second group (holo-cytoplasts), nuclei were removed with a minimum volume of cytoplasm (Fig. 3G). Some oocytes that had been manipulated in the same manner to remove the same volume of cytoplasm without removing the nuclei were used as sham-operated oocytes (holo-oocytes). Figure 4 is a schematic in which the experimental design is summarized.

\section{Statistical analysis}

Data were analyzed using a $\chi^{2}$ test (SPSS 13.0; SPSS Inc., Chicago, IL, USA). $P$ values $<0.05$ were considered to indicate statistical significance.

\section{Acknowledgements}

This research was supported by the Special Funds for Major State Basic Research Projects (Grant number: 2001CB509905). We are grateful to Ms Xiang-Fen Song, Ms Shi-Wen Li, Dr ChangLong Nan, Dr Shen Yin, Dr Sheng-Li Lin, and Dr Bo Xiong for their technical assistance. The authors declare that there is no 
conflict of interest that would prejudice the impartiality of this scientific work.

\section{References}

Afshar K, Barton NR, Hawley RS \& Goldstein LS 1995 DNA binding and meiotic chromosomal localization of the Drosophila nod kinesin-like protein. Cell 81 129-138.

Balakier H \& Tarkowski AK 1980 The role of germinal vesicle karyoplasm in the development of male pronucleus in the mouse. Experimental Cell Research 128 79-85.

Brunet S, Polanski Z, Verlhac MH, Kubiak JZ \& Maro B 1998 Bipolar meiotic spindle formation without chromatin. Current Biology $\mathbf{8}$ 1231-1234.

Bucciarelli E, Giansanti MG, Bonaccorsi S \& Gatti M 2003 Spindle assembly and cytokinesis in the absence of chromosomes during Drosophila male meiosis. Journal of Cell Biology 160 993-999.

Calarco PG, Donahue RP \& Szollosi D 1972 Germinal vesicle breakdown in the mouse oocyte. Journal of Cell Science 10 369-385.

Calarco-Gillam PD, Siebert MC, Hubble R, Mitchison T \& Kirschner M 1983 Centrosome development in early mouse embryos as defined by an autoantibody against pericentriolar material. Cell 35 621-629.

Carazo-Salas RE, Guarguaglini G, Gruss OJ, Segref A, Karsenti E \& Mattaj IW 1999 Generation of GTP-bound Ran by RCC1 is required for chromatin-induced mitotic spindle formation. Nature 400 178-181.

Carazo-Salas RE, Gruss OJ, Mattaj IW \& Karsenti E 2001 Ran-GTP coordinates regulation of microtubule nucleation and dynamics during mitotic-spindle assembly. Nature Cell Biology 3 228-234.

Caudron M, Bunt G, Bastiaens P \& Karsenti E 2005 Spatial coordination of spindle assembly by chromosome-mediated signaling gradients. Science 309 1373-1376.

Van Cauwenberge A \& Alexandre H 1992 Control of microtubule nucleating activity in the cytoplasm of maturing mouse oocytes. International Journal of Developmental Biology 36 143-150.

Chang CC, Nagy ZP, Abdelmassih R, Yang X \& Tian XC 2004 Nuclear and microtubule dynamics of G2/M somatic nuclei during haploidization in germinal vesicle-stage mouse oocytes. Biology of Reproduction $\mathbf{7 0}$ 752-758.

Compton DA 2000 Spindle assembly in animal cells. Annual Review of Biochemistry 69 95-114.

Dogterom M, Felix MA, Guet CC \& Leibler S 1996 Influence of M-phase chromatin on the anisotropy of microtubule asters. Journal of Cell Biology 133 125-140.

Dumont J, Petri S, Pellegrin F, Terret ME, Bohnsack MT, Rassinier P, Georget V, Kalab P, Gruss OJ \& Verlhac MH 2007 A centriole- and RanGTP-independent spindle assembly pathway in meiosis I of vertebrate oocytes. Journal of Cell Biology 176 295-305.

Gaetz J, Gueroui Z, Libchaber A \& Kapoor TM 2006 Examining how the spatial organization of chromatin signals influences metaphase spindle assembly. Nature Cell Biology 8 924-932.

Gueth-Hallonet C, Antony C, Aghion J, Santa-Maria A, Lajoie-Mazenc I, Wright M \& Maro B $1993 \gamma$-Tubulin is present in acentriolar MTOCs during early mouse development. Journal of Cell Science 105 157-166.

Karsenti E \& Vernos I 2001 The mitotic spindle: a self-made machine. Science 294 543-547.

Karsenti E, Newport J, Hubble R \& Kirschner M 1984 Interconversion of metaphase and interphase microtubule arrays, as studied by the injection of centrosomes and nuclei into Xenopus eggs. Journal of Cell Biology 98 1730-1745.

Katagiri C \& Moriya M 1976 Spermatozoan response to the toad egg matured after removal of germinal vesicle. Developmental Biology $\mathbf{5 0}$ 235-241.

Maro B, Howlett SK \& Webb M 1985 Non-spindle microtubule organizing centers in metaphase II-arrested mouse oocytes. Journal of Cell Biology 101 1665-1672.

McKim KS \& Hawley RS 1995 Chromosomal control of meiotic cell division. Science 270 1595-1601.
Meng XQ, Fan HY, Zhong ZS, Zhang G, Li YL, Chen DY \& Sun QY 2004 Localization of gamma-tubulin in mouse eggs during meiotic maturation, fertilization, and early embryonic development. Journal of Reproduction and Development 50 97-105.

Mitchison TJ \& Salmon ED 2001 Mitosis: a history of division. Nature Cell Biology 3 E17-E21.

Murphy TD \& Karpen GH 1995 Interactions between the nod + kinesin-like gene and extracentromeric sequences are required for transmission of a Drosophila minichromosome. Cell 81 139-148.

Nicklas RB \& Gordon GW 1985 The total length of spindle microtubules depends on the number of chromosomes present. Journal of Cell Biology $1001-7$.

Palacios MJ, Joshi HC, Simerly C \& Schatten G 1993 Gamma-tubulin reorganization during mouse fertilization and early development. Journal of Cell Science 104 383-389.

de Saint Phalle B \& Sullivan W 1998 Spindle assembly and mitosis without centrosomes in parthenogenetic Sciara embryos. Journal of Cell Biology 141 1383-1391.

Schultz RM, Letourneau GE \& Wassarman PM 1978 Meiotic maturation of mouse oocytes in vitro: protein synthesis in nucleate and anucleate oocyte fragments. Journal of Cell Science 30 251-264.

Smirnova EA \& Bajer AS 1992 Spindle poles in higher plant mitosis. Cell Motility and the Cytoskeleton 23 1-7.

Steffen W, Fuge H, Dietz R, Bastmeyer M \& Muller G 1986 Aster-free spindle poles in insect spermatocytes: evidence for chromosomeinduced spindle formation? Journal of Cell Biology 102 1679-1687.

Sun QY, Lai L, Wu GM, Park KW, Day BN, Prather RS \& Schatten H 2001 Microtubule assembly after treatment of pig oocytes with taxol: correlation with chromosomes, gamma-tubulin, and MAP kinase. Molecular Reproduction and Development 60 481-490.

Szollosi D, Calarco P \& Donahue RP 1972 Absence of centrioles in the first and second meiotic spindles of mouse oocytes. Journal of Cell Science 11 521-541.

Takeuchi T, Ergun B, Huang TH, Rosenwaks Z \& Palermo GD 1999 A reliable technique of nuclear transplantation for immature mammalian oocytes. Human Reproduction 14 1312-1317.

Theurkauf WE \& Hawley RS 1992 Meiotic spindle assembly in Drosophila females: behavior of nonexchange chromosomes and the effects of mutations in the nod kinesin-like protein. Journal of Cell Biology 116 1167-1180.

Verlhac MH, de Pennart H, Maro B, Cobb MH \& Clarke HJ 1993 MAP kinase becomes stably activated at metaphase and is associated with microtubule-organizing centers during meiotic maturation of mouse oocytes. Developmental Biology 158 330-340.

Vernos I, Raats J, Hirano T, Heasman J, Karsenti E \& Wylie C 1995 Xklp1, a chromosomal Xenopus kinesin-like protein essential for spindle organization and chromosome positioning. Cell 81 117-127.

Wang SZ \& Adler R 1995 Chromokinesin: a DNA-binding, kinesin-like nuclear protein. Journal of Cell Biology 128 761-768.

Winey M \& O'Toole ET 2001 The spindle cycle in budding yeast. Nature Cell Biology 3 E23-E27.

Zhang D \& Nicklas RB 1995 Chromosomes initiate spindle assembly upon experimental dissolution of the nuclear envelope in grasshopper spermatocytes. Journal of Cell Biology 131 1125-1131.

Zheng Y, Wong ML, Alberts B \& Mitchison T 1995 Nucleation of microtubule assembly by a gamma-tubulin-containing ring complex. Nature 378 578-583.

Received 2 April 2007

First decision 3 May 2007

Revised manuscript received 30 August 2007

Accepted 24 September 2007 